\title{
Reactions of $\mathrm{Ti}, \mathrm{V}, \mathrm{Nb}$ and Ta with $\mathrm{N}_{2}$ Gas at $2000^{\circ} \mathrm{C}$ under Concentrated Solar Beam in a Solar Furnace at PROMES-CNRS
}

\author{
Nobumitsu Shohoji ${ }^{1, *}$, Fernando Almeida Costa Oliveira ${ }^{1}$, Luís Guerra Rosa ${ }^{2}$, \\ Gilles Peraudeau ${ }^{3}$, Bernard Granier $^{3}$, Teresa Magalhães ${ }^{1}$ and Jorge Cruz Fernandes ${ }^{2}$ \\ ${ }^{1}$ LNEG-Laboratório Nacional de Energia e Geologia, LEN-Laboratório de Energia, \\ Estrada do Paço do Lumiar, 22, 1649-038 Lisboa, Portugal \\ ${ }^{2}$ IST-Instituto Superior Técnico, Departamento de Engenharia Mecânica, Av. Rovisco Pais, 1049-001 Lisboa, Portugal \\ ${ }^{3}$ PROMES-CNRS, Laboratoire Procédés, Matériaux et Energie Solaire, 7, rue du Four Solaire, Odeillo 66120, France
}

Reactions of $d$-group transition metals including Ti, $\mathrm{V}, \mathrm{Nb}$ and Ta with $\mathrm{N}_{2}$ gas were investigated at target temperature of $2000^{\circ} \mathrm{C}$ under heating with concentrated solar beam at PROMES-CNRS in Odeillo (France). In the typical experimental setup using graphite crucible as the sample holder, synthesis of pure nitride with $f c c$ (face centred cubic) structure was proved to be difficult on account of inevitable $\mathrm{C}_{2}$ radical plume with high carbon activity $a(\mathrm{C})$ from the graphite components and the majority of reaction products were carbo-nitride. By insertion of Sky blue filter in the solar beam path before entering into the parabolic concentrator, $\mathrm{C}_{2}$ radical formation appeared to be efficiently suppressed and phases with high $\mathrm{N}$ content were synthesized. However, under conditions in which $\mathrm{C}_{2}$ radical plume yield was considerable, nitrogen content in the reaction products became suppressed and tetragonal $\mathrm{Nb}_{4} \mathrm{~N}_{3}$ yielded in place of $f c c \mathrm{NbN}$ from $\mathrm{Nb}$ or $\mathrm{N}$-deficit sub-nitride Ta $2 \mathrm{~N}$ in place of mono-nitride TaN formed. [doi:10.2320/matertrans.M2010314]

(Received September 10, 2010; Accepted December 28, 2010; Published February 9, 2011)

Keywords: solar furnace, colour filter, carbo-nitride, titanium, vanadium, niobium, tantalum, tetragonal lattice distortion

\section{Introduction}

Since 1997, we have been using a solar furnace at PSA (Plataforma Solar de Almería) as the heat source for synthesizing carbides of $d$-group transition elements. ${ }^{1-10)}$ This line of work was continued after 2005 using a solar furnace at PROMES-CNRS (Laboratoire Procédés, Matériaux et Energie Solaire) in Odeillo. ${ }^{11-18)}$

During the course of these works, we managed to demonstrate that the solar furnaces are capable of concentrating the solar energy by a factor $10^{4}$ or higher to a spot size of diameter, $5 \mathrm{~cm}$ (PSA) or $1 \mathrm{~cm}$ (PROMES-CNRS), and therefore can be used as the ecological renewable heat source to trigger high temperature reactions leading to refractory carbide synthesis.

Although our main target of this series of works done using solar furnace at PSA (Spain) $)^{1-10)}$ and that at PROMES-CNRS (France) ${ }^{11-18)}$ were carbide synthesis, brief comparative test runs were undertaken to check influence of $\mathrm{N}_{2}$ gas environment compared with the standard inert Ar gas atmosphere ${ }^{1,2)}$ on the carbide formation reaction kinetics for $\mathrm{M}=\mathrm{Si}$ and $\mathrm{W}$. Our observation of the XRD (X-ray diffraction) evidences in these works showed no sign of nitride or carbo-nitride formation for neither $\mathrm{Si}$ nor $\mathrm{W}$ but certain extent of retardation for carbide formation reaction was detected for $\mathrm{Si}$ and it was interpreted as the competing nitriding reaction to form $\mathrm{Si}_{3} \mathrm{~N}_{4}$ against carbide forming reaction to yield $\mathrm{SiC}$ in $\mathrm{N}_{2}$ gas environment. On the other hand, no such kinetic effect of $\mathrm{N}_{2}$ gas environment was detected for $\mathrm{W}$ and it was appreciated as representing the incomparably strong tendency of $\mathrm{W}$ towards carbide formation than towards nitride formation.

*Corresponding author, E-mail: nobumitsu.shohoji@lneg.pt
In the present work, nitride synthesis was attempted in a solar furnace at PROMES-CNRS with the target temperature set at $2000^{\circ} \mathrm{C}$ in $\mathrm{N}_{2}$ gas environment for $\mathrm{M}=\mathrm{Ti}, \mathrm{V}, \mathrm{Nb}$ and Ta.

\section{Experimental}

\subsection{Sample materials}

Specifications of the sample materials used in the present work were as follows;

Ti: $99 \%$ pure supplied from Ventron (Karlsruhe, Germany) with grain size $<45 \mu \mathrm{m}$.

$\mathrm{V}$ : $99 \%$ pure supplied from Goodfellow (Cambridge, England) with grain size $<45 \mu \mathrm{m}$,

$\mathrm{Nb}$ : $99.5 \%$ pure supplied from L. Light (Cambridge, England) with grain size $<125 \mu \mathrm{m}$,

Ta: $99.9 \%$ pure supplied from Goodfellow (Cambridge, England) with grain size $<75 \mu \mathrm{m}$.

$\mathrm{N}_{2}$ gas was $99.99 \%$ pure (Azote $\mathrm{U}$ ) supplied from Air Liquide (France) with nominal impurities, $\mathrm{H}_{2} \leq 5 \mathrm{ppm}$ and $\mathrm{O}_{2} \leq 5$ ppm.

Each test piece was a pellet of dimension, $10 \mathrm{~mm} \phi$ and about $4 \mathrm{~mm}$ thick, prepared by compacting the as-supplied powders by applying uniaxial pressure of $400 \mathrm{MPa}$ at ambient temperature.

\subsection{New solar experimental setup at PROMES-CNRS}

The PROMES solar furnace used for this work was consisted of a parabolic mirror with focal length $851 \mathrm{~mm}$ and diameter $200 \mathrm{~cm}$ (the cone tip angle $120^{\circ}$ ) receiving solar beams reflected by a flat mirror beneath. The flat mirror reflector was mobile along NS (north-south) and EW (eastwest) axes driven by servo electric motor system to track the sun. The effective power of the solar furnace was $2 \mathrm{~kW}$ with a peak density at the focal point $16 \mathrm{MW} / \mathrm{m}^{2}$. 


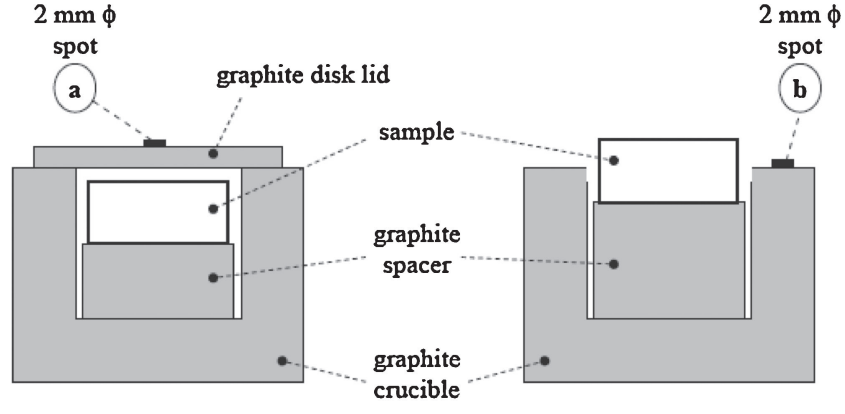

Setup A

Setup B

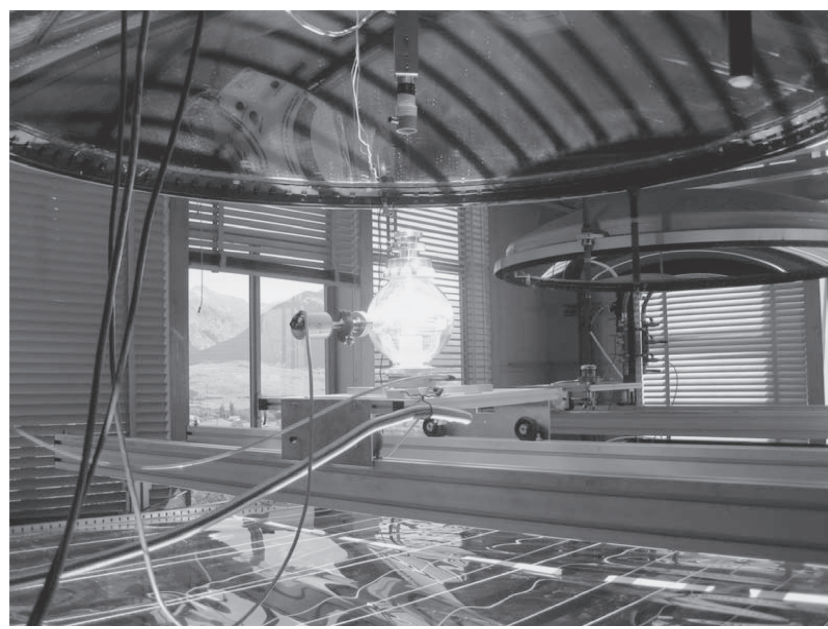

Fig. 1 Graphite crucible setups and general appearance of the experimental configuration with filter. Top left: Setup A with graphite lid, pyrometric temperature measurement done at spot (a), Top right: Setup B without lid, pyrometric temperature measurement done at spot (b). Bottom: General appearance of the experimental configuration showing parabolic concentrator of $2 \mathrm{~m} \phi$ at the top, $3 l$ Pyrex glass reaction chamber at the center and yellow filter of dimension $2 \times 2 \mathrm{~m}$ at the bottom. Stripes seen over the parabolic concentrator surface represents the shades of louvered shutters placed beneath the filter. Optical Pyrometer is hanged from the center of the parabolic concentrator looking down straight to the crucible.

The detailed experimental setup used for the series of works done at PROMES-CNRS between 2005 and 2008 was given in the earlier publications. ${ }^{11-18)}$ In the present experimental runs carried out between 28th June 2010 and 15th July 2010, there were several modifications introduced in the experimental setup.

For convenience of the readers, general appearance of the experimental configuration is presented in Fig. 1 together with two graphite crucible setup modes employed in the experiments.

In the following, modifications introduced in the new setup and resultant alterations in experimental procedure are described by comparing with the previous ones.

The first modification was the used parabolic mirror as described above. In the present test runs, $\phi 200 \mathrm{~cm}$ parabola was used whereas, in the preceding test runs, the mirror diameter was $\phi 150 \mathrm{~cm}$ with focal length $648 \mathrm{~mm}$ and nominal power $1.5 \mathrm{~kW}$.

The second alteration was the Pyrex glass reaction chamber capacity; about $3 l$ in the present test runs compared with $5 l$ in the preceding series of works. ${ }^{11-18)}$
The third change was the introduction of the louvered shutter made of light-weight $\mathrm{C} / \mathrm{C}$ fiber composite sheet to control the amount of solar beam entering to the parabolic concentrator. By introduction of the shutter, reaction chamber height was fixed to make the test piece top surface at the exact focal spot of the concentrated solar beam and the heating process was controlled by stepwise opening of the shutter (shutter opening angle $0^{\circ}$ refers to the complete closure and $90^{\circ}$ the full opening). In the earlier setup used for the experiments done between 2005 and 2008, ${ }^{1-18)}$ target temperature was controlled by adjusting the height of the test piece top surface at certain extent of downward de-focusing from the exact focal spot of the solar beam and the reaction chamber was brought to this location by sliding over a pair of guiding rails manually.

The fourth modification was the configuration for optical one-colour pyrometer temperature measurement. In the previous setting, temperature was measured from the side of the graphite crucible with triple vertical slits of width $1 \mathrm{~mm}$ arranged to realize condition closer to the black body radiation condition. ${ }^{11-18)}$ In the new configuration, optical pyrometer (HEITRONICS infra-red pyrometer Model KT15; single-colour at wavelength $\lambda=5 \mu \mathrm{m}$ ) set at the center of the parabolic concentrator was looking down the reaction chamber and the measured pyrometric temperature was displayed in real-time over the display of the PC (personal computer) through EasyConfig software with the calibrated emissivity $\varepsilon$ for graphite taken to be 0.70 at $\lambda=5 \mu \mathrm{m}$. This software displayed graphically the instantaneous temperature profile against time over each $30 \mathrm{~s}$ duration and registered the measured maximum temperature throughout the single monitoring session taking into account the following factors; (i) light transmittance $\tau$ of the fluorine glass window was taken to be 0.92 on the basis of preliminary measurements using a black body cavity,

(ii) the values of graphite surface emissivity $\varepsilon$ at wavelengths, $0.7 \mu \mathrm{m}$ and $5 \mu \mathrm{m}$, as reported by Touloukian and Dewitt. ${ }^{19)}$

The EasyConfig software possessed no capacity to store the measurement record.

Corresponding to these modifications of the experimental setup in the solar furnace, solar irradiation procedures were somewhat altered from those in the earlier works. ${ }^{11-18)}$

\subsection{Solar irradiation procedures}

As the consequence of introduced modifications to the solar furnace system configuration as well as of nature of the starting materials used in the experiments, experimental procedures were modified from those in the preceding series of works ${ }^{1-18)}$ to the ones described below.

In the preceding works, ${ }^{1-18)} \mathrm{M}$ powders mixed with carbon materials (graphite $\mathrm{G}$ or amorphous carbon $\mathrm{aC}$ ) compacted into form of pellet were used as the starting material. Thence, even with the extremely high rate of heating at the onset of solar radiation to reach a set target temperature from the ambient temperature directly, passive oxide film inevitably present over $M$ powder surface must have instantaneously reacted with the tightly compacted $\mathrm{C}$ against the $\mathrm{M}$ powder surface to be evaporated in form of $\mathrm{CO}$ or $\mathrm{CO}_{2}$ to expose clean $\mathrm{M}$ substrate allowing subsequent carbide forming reaction. 
Table 1 Grouped test runs with measured test piece weight and $\mathrm{N}_{2}$ gas pressure in the reaction chamber of about $3000 \mathrm{~m} l$.

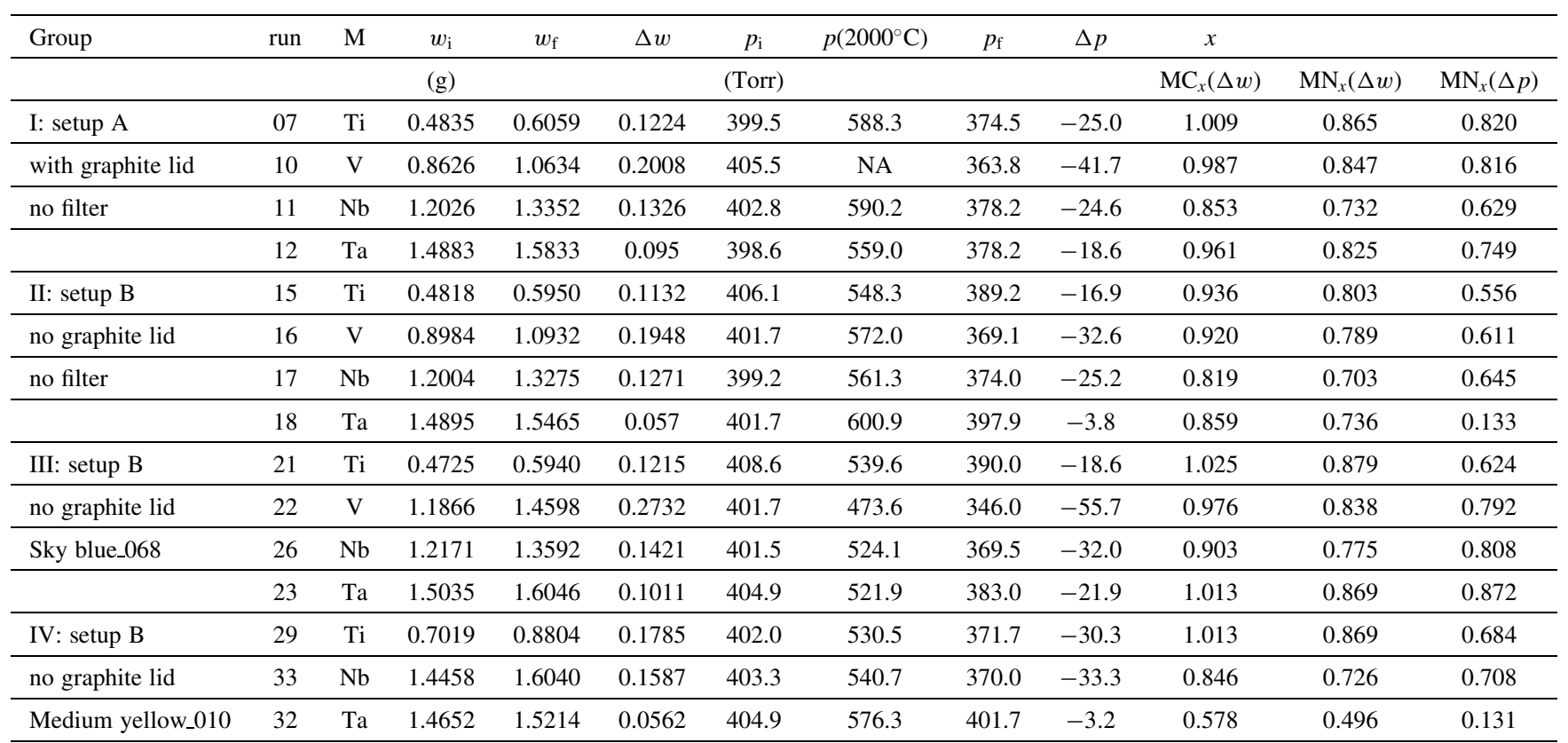

$w_{\mathrm{i}}$ : initial test piece weight $(\mathrm{g})$,

$w_{\mathrm{f}}$ : final test piece weight $(\mathrm{g})$,

$\Delta w=w_{\mathrm{f}}-w_{\mathrm{i}}$,

$p_{\mathrm{i}}$ : initial $\mathrm{N}_{2}$ gas pressure (Torr)

$p\left(2000^{\circ} \mathrm{C}\right)$ : maximum measured $\mathrm{N}_{2}$ gas pressure at the reaction temperature (Torr),

$p_{\mathrm{f}}$ : final $\mathrm{N}_{2}$ gas pressure (Torr),

$\Delta p=p_{\mathrm{f}}-p_{\mathrm{i}}$,

$\mathrm{MC}_{x}(\Delta w)$ : estimated $\mathrm{MC}_{x}$ from $\Delta w$ assuming the measured mass gain was due to full conversion to $\mathrm{MC}_{x}$,

$\mathrm{MN}_{x}(\Delta w)$ : estimated $\mathrm{MN}_{x}$ from $\Delta w$ assuming the measured mass gain was due to full conversion to $\mathrm{MN}_{x}$,

$\mathrm{MN}_{x}(\Delta p)$ : estimated $\mathrm{MN}_{x}$ from $\Delta p$.

note 1) evaporation weight loss by sublimation of $\mathrm{M}$ during solar heating was not taken into account in the estimation of $\mathrm{MC}_{x}$ nor $\mathrm{MN}_{x}$.

note 2) absorpbed amount of $\mathrm{N}$ atoms into the sample material, $\Delta n(\mathrm{~mol})$, in the estimation of $\mathrm{MN}_{x}(\Delta p)$ using the relationship

$\Delta n(\mathrm{~mol})=2 \cdot[\Delta p \cdot V /(R \cdot T)] \approx 0.000331 \cdot \Delta p$ (Torr)

assuming $T=300 \mathrm{~K}$ and $V=3096 \mathrm{ml}$.

On the other hand, in the present experiments, starting material was compacted $\mathrm{M}$ powders alone. Some of $\mathrm{M}$ materials seemed to be with comparatively high equilibrium vapor pressure according to available thermodynamic data. ${ }^{20)}$ Thus, excessively high heating rate at the onset of the solar heating was concerned to result in unfavourable sublimation loss of $\mathrm{M}$ during the initial stage of heating before it is converted to nitride. To avoid this potential risk, initial heating was done stepwise after opening the louvered shutter to $35^{\circ}$ at the beginning. This initial shutter opening led to pyrometric temperature reading of around $1000^{\circ} \mathrm{C}$. At this initial stage, absorption of $\mathrm{N}_{2}$ gas into the test piece usually started judging from the time variation pattern of the pressure gauge reading at a stabilized temperature. Thereafter, shutter opening was increased stepwise checking the $\mathrm{N}_{2}$ pressure variation pattern of the reaction chamber to $75^{\circ}$. At this shutter opening, pyrometer reading reached at around $2000^{\circ} \mathrm{C}$ for the setup A depicted in Fig. 1 under the natural solar intensity range $900 \sim 1000 \mathrm{~W} / \mathrm{m}^{2}$. The experiments with the setup A with graphite lid were done in order to determine the degree of the shutter opening to reach the target temperature $2000^{\circ} \mathrm{C}$.

As anticipated in Fig. 1, actual temperature of the test piece top surface temperature must have been appreciably lower than $2000^{\circ} \mathrm{C}$.
In the experiments with the setup B with no graphite lid, test piece top surface was arranged to be about the same level as the graphite lid surface in the setup A by adjusting the graphite spacer thickness below the test piece. By so doing, the test piece top surface temperature was assumed to reach the target temperature $2000^{\circ} \mathrm{C}$ in the experiments with the setup B with no graphite lid. For the experiments with the setup $\mathrm{B}$, pyrometer temperature monitoring was done at the spot (b) in place of (a) in the setup A. The measured pyrometric temperature at spot (b) was by about $300 \mathrm{~K}$ lower than that at spot (a). This was not surprising noting that the hot spot size at the focal point of the used solar furnace was about $\phi 10 \mathrm{~mm}$ just covering the test piece diameter.

The high purity $\mathrm{N}_{2}$ gas was introduced into the reaction chamber to partial pressure $p\left(\mathrm{~N}_{2}\right) \approx 400$ Torr $(\approx 55 \mathrm{kPa})$ at ambient temperature after at least twice evacuating and flushing with the same high purity $\mathrm{N}_{2}$ gas.

Each test piece was held at the target temperature for $30 \mathrm{~min}$.

\subsection{Measured experimental parameters}

Measured experimental parameter values and calculated parameter values on the basis of the experimental parameter values are listed in Table 1. 
Initial mass $w_{\mathrm{i}}(\mathrm{g})$ as well as the final mass $w_{\mathrm{f}}(\mathrm{g})$ was measured for each test piece and, from the calculated mass gain $\Delta w\left(=w_{\mathrm{f}}-w_{\mathrm{i}}\right)$, very crude estimation was made for the composition $x$ of the reaction product assuming that the compound was pure $\mathrm{MC}_{x}, \mathrm{MC}_{x}(\Delta w)$, or pure $\mathrm{MN}_{x}$, $\mathrm{MN}_{x}(\Delta w)$. The $x$ values evaluated as such were definitely over-estimate noting that certain extent of sublimation loss of the metal constituent was inevitable during heating under concentrated solar beam.

As seen in Table 1, initial nitrogen pressure $p_{\mathrm{i}}$ in the reaction chamber at ambient temperature was controlled to be at around $400 \pm 5$ Torr. At the reaction temperature of about $2000^{\circ} \mathrm{C}$, the measured nitrogen partial pressure $p\left(2000^{\circ} \mathrm{C}\right)$ reached to a level between 600 Torr (run 18 for Ta) and 475 Torr (run 22 for $\mathrm{V}$ ) depending on the amount of $\mathrm{N}_{2}$ gas absorbed into the test piece. The final nitrogen pressure $p_{\mathrm{f}}$ at ambient temperature immediately before opening the reaction chamber was also registered to estimate the composition $x$ in $\mathrm{MN}_{x}$ from the calculated $\Delta p$ $\left(=p_{\mathrm{f}}-p_{\mathrm{i}}\right)$. The $x$ evaluated as such must be also overestimation noting the inevitable sublimation loss of the metal constituent. The most of the estimated $x$ for $\mathrm{MN}_{x}(\Delta p)$ fell in the range between 0.82 (run 07 for Ti) and 0.56 (run 15 for $\mathrm{Ti}$ ) while that for some Ta specimens ( 0.13 for run 18 and run 32) was considerably low.

\section{Results and Discussion}

Before presenting experimental evidences in detail, essential aspects of carbides, carbo-nitrides and nitrides of $\mathrm{Ti}, \mathrm{V}, \mathrm{Nb}$ and $\mathrm{Ta}$ are reviewed because, in solar heating experiments to reach temperature exceeding $1500^{\circ} \mathrm{C}$, sample holder is typically made of graphite and influence of $\mathrm{C}$ on the reaction product is inevitable.

Under condition of solar heating, $\mathrm{C}_{2}$ radical plume was reported to yield from graphite block. ${ }^{21)}$

Thus, prior to starting this series of experiments targeting nitride synthesis in solar furnace, we reviewed some characteristics of carbo-nitrides of $\mathrm{Ti}, \mathrm{V}, \mathrm{Nb}$ and $\mathrm{Ta}$. In the following, essential features of the experimental evidences are described.

\subsection{Characteristic features of carbo-nitrides of $\mathrm{Ti}, \mathrm{V}, \mathrm{Nb}$ and Ta}

Carbides and nitrides of $d$-group transition elements including $\mathrm{Ti}, \mathrm{V}, \mathrm{Nb}$ and $\mathrm{Ta}$ are characterized by their extensive range of non-stoichiometric composition. When we express these compounds in form of $\mathrm{MX}_{x}(\mathrm{X}=\mathrm{C}, \mathrm{N})$, composition $x(=\mathrm{X} / \mathrm{M})$ in so-called mono-carbide or mononitride might vary between 0.5 to 1 . Correspondingly, lattice constant values of a given carbide or nitride vary widely depending on $x$.

Table 2 summarizes the calculated values of high $(h k l)$ index XRD peak positions with $\mathrm{Cu} K \alpha$ radiation for carbides and nitrides of $\mathrm{Ti}, \mathrm{V}, \mathrm{Nb}$ and $\mathrm{Ta}$, on the basis of available literature data. ${ }^{22-26)}$

This inherent nature of extensive homogeneity composition ranges for carbides and nitrides of $\mathrm{Ti}, \mathrm{V}, \mathrm{Nb}$ and $\mathrm{Ta}$ makes the XRD characterization of the synthesized carbonitride difficult although we present the possible lattice

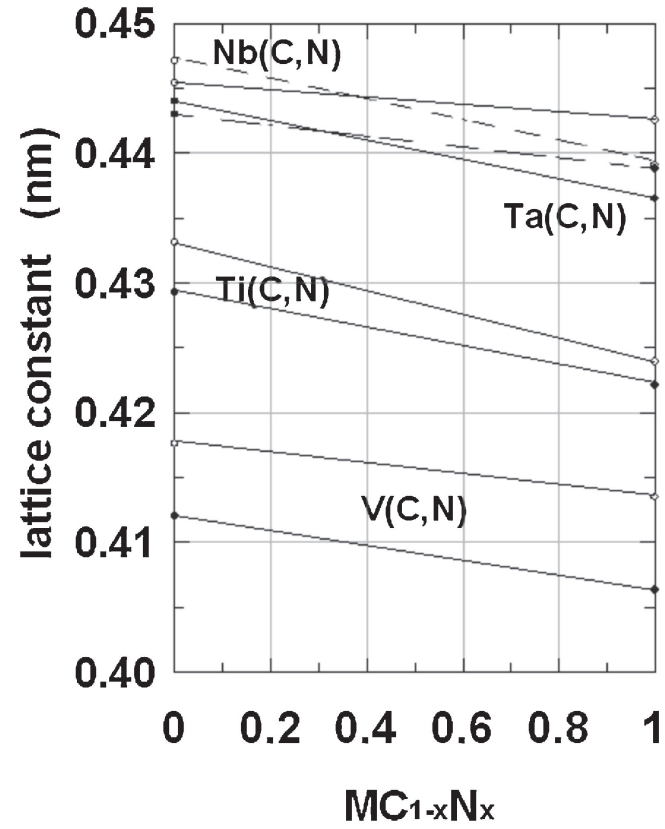

Fig. 2 Ranges of lattice constant $a(\mathrm{~nm})$ for $f c c \mathrm{M}(\mathrm{C}, \mathrm{N})$ phases under consideration $(\mathrm{M}=\mathrm{Ti}, \mathrm{V}, \mathrm{Nb}$ and $\mathrm{Ta})$. See Table 2 for data sources for $a(\mathrm{MC})$ and $a(\mathrm{MN})$.

constant vs. carbo-nitride composition relationship as depicted in Fig. 2 for convenience. In Fig. 2, carbo-nitride composition on the horizontal axis is given as $\mathrm{MC}_{1-x} \mathrm{~N}_{x}$ but, rigorously speaking, it must be presented as $\mathrm{MC}_{\mathrm{p}} \mathrm{N}_{\mathrm{q}}$ $(0.5<p+q<1)$.

Anyway, we can take the shift of $2 \theta_{\mathrm{hkl}}$ towards the higher angle side as the rough index for increased $\mathrm{N}$ content in the carbo-nitride synthesized.

\subsection{Acquired XRD patterns for the top and the bottom surfaces}

The acquired XRD patterns over $2 \theta=90 \sim 145^{\circ}$ for the top and the bottom surfaces are summarized in Figs. 3 (Ti), $4(\mathrm{~V}), 5(\mathrm{Nb})$ and $6(\mathrm{Ta})$.

It is interesting to note that, in the Group I experiments undertaken with graphite lid, extent of carburization was generally higher at the top surface than at the bottom surface; compare (a) and (b) in Figs. 3-5.

In Fig. 3 for $\mathrm{M}=\mathrm{Ti}$, four peaks with indices, (331), (420), (422) and (511/333), are seen on going from the lower $2 \theta$ to the higher 2 $\theta$. In Figs. 3(a) and (b), each peak splits into two; the lower one representing $\mathrm{TiC}_{1-x} \mathrm{~N}_{x}$ with higher $\mathrm{C}$ content and the higher one that with higher N. Comparing (a) and (b) in Fig. 3, it is evident that the relative intensity of the higher sub-peak corresponding to the same $(h k l)$ index was higher in (b) representing the bottom surface than in (a) representing the top surface of the same test piece.

In Fig. 4 for $M=V$, splitting of each peak was not detected but, when comparing the XRD patterns between (a) and (b), the peak with the same $(h k l)$ index was more on the higher $2 \theta$ side for (b) than for (a) suggesting that $x$ in $\mathrm{MC}_{1-x} \mathrm{~N}_{x}$ at the bottom surface (b) was higher than at the top surface (a).

These evidences suggest that the carbon activity $a(\mathrm{C})$ in the environment was higher at the top surface surrounding 
Table 2 Calculated XRD diffraction angles $2 \theta_{\mathrm{hkl}}$ with $\mathrm{Cu} K \alpha$ radiation $(\lambda(\mathrm{Cu} K \alpha)=0.1541838 \mathrm{~nm})$ for $f c c$ (face centred cubic) nonstoichiometric $\mathrm{MC}_{x}$ and $\mathrm{MN}_{x}$ phases under consideration in the range of $2 \theta_{\mathrm{hkl}}$ between $95^{\circ}$ and $145^{\circ}$ from the reported values of the maximum and the minimum lattice constant values, $a_{\max }$ and $a_{\min }$.

\begin{tabular}{|c|c|c|c|c|c|}
\hline \multirow[t]{2}{*}{$\mathrm{M}$} & \multirow[t]{2}{*}{$h k l$} & \multicolumn{4}{|l|}{$2 \theta_{\mathrm{hkl}}($ degree $)$} \\
\hline & & $\mathrm{MC}_{x}$ & & $\mathrm{MN}_{x}$ & \\
\hline \multirow[t]{5}{*}{$\mathrm{Ti}$} & & $\begin{array}{c}a_{\max }=0.4331 \mathrm{~nm} \\
\text { at } x=0.85\end{array}$ & $\begin{array}{c}a_{\min }=0.4293 \mathrm{~nm} \\
\text { at } x=0.55\end{array}$ & $\begin{array}{c}a_{\max }=0.4239 \mathrm{~nm} \\
\text { at } x=0.999\end{array}$ & $\begin{array}{c}a_{\min }=0.4221 \mathrm{~nm} \\
\text { at } x=0.605\end{array}$ \\
\hline & 331 & 101.77 & 103.03 & 104.88 & 105.52 \\
\hline & 420 & 105.51 & 106.87 & 108.84 & 109.53 \\
\hline & 422 & 121.39 & 123.25 & 125.98 & 126.95 \\
\hline & $511 / 333$ & 135.31 & 137.89 & 141.81 & 143.25 \\
\hline \multirow[t]{4}{*}{$\mathrm{V}$} & & $\begin{array}{c}a_{\max }=0.4167 \mathrm{~nm} \\
\text { at } x=0.9\end{array}$ & $\begin{array}{c}a_{\min }=0.4120 \mathrm{~nm} \\
\text { at } x=0.7\end{array}$ & $\begin{array}{c}a_{\max }=0.4135 \mathrm{~nm} \\
\text { at } x=0.996\end{array}$ & $\begin{array}{c}a_{\min }=0.4063 \mathrm{~nm} \\
\text { at } x=0.707\end{array}$ \\
\hline & $411 / 330$ & 103.43 & 105.10 & 104.56 & 107.22 \\
\hline & 331 & 107.49 & 109.28 & 108.72 & 111.56 \\
\hline & 420 & 111.65 & 113.59 & 112.98 & 116.11 \\
\hline \multirow[t]{5}{*}{$\mathrm{Nb}$} & & $\begin{array}{c}a_{\max }=0.4471 \mathrm{~nm} \\
\text { at } x=1\end{array}$ & $\begin{array}{c}a_{\min }=0.4430 \mathrm{~nm} \\
\text { at } x=0.7\end{array}$ & $\begin{array}{c}a_{\max }=0.4391 \mathrm{~nm} \\
\text { at } x=0.975\end{array}$ & $\begin{array}{c}a_{\min }=0.4388 \mathrm{~nm} \\
\text { at } x=0.924\end{array}$ \\
\hline & 331 & 98.16 & 98.67 & 99.87 & 99.96 \\
\hline & 420 & 101.70 & 102.18 & 103.47 & 103.57 \\
\hline & 422 & 116.31 & 116.95 & 118.66 & 118.79 \\
\hline & $511 / 333$ & 128.58 & 129.41 & 131.65 & 131.82 \\
\hline \multirow[t]{5}{*}{$\mathrm{Ta}$} & & $\begin{array}{c}a_{\max }=0.4454 \mathrm{~nm} \\
\text { at } x=0.97\end{array}$ & $\begin{array}{c}a_{\min }=0.4440 \mathrm{~nm} \\
\text { at } x=0.71\end{array}$ & $\begin{array}{c}a_{\max }=0.4425 \mathrm{~nm} \\
\text { at } x=?\end{array}$ & $\begin{array}{c}a_{\min }=0.4365 \mathrm{~nm} \\
\text { at } x=?\end{array}$ \\
\hline & 331 & 97.96 & 98.37 & 98.82 & 100.68 \\
\hline & 420 & 101.45 & 101.88 & 102.36 & 104.33 \\
\hline & 422 & 115.99 & 116.56 & 117.19 & 119.80 \\
\hline & $511 / 333$ & 128.17 & 128.90 & 129.72 & 133.17 \\
\hline
\end{tabular}

= lattice constant values for $\mathrm{MC}_{x}$ from Storms. ${ }^{22)}$

$=$ lattice constant values for $\mathrm{TiN}_{x}$ from Nagakura et al. ${ }^{23)}$

$a\left(\operatorname{TiN}_{x}\right)=0.41925+0.00467 x(\mathrm{~nm}),(0.605<x<0.999)$.

$=$ lattice constant values for $\mathrm{VN}_{x}$ from Langauer and Ettmayer: ${ }^{24)}$

$a\left(\mathrm{VN}_{x}\right)=0.3887+0.02488 x-(1.083 \pm 0.021) \cdot 10^{-4} T^{1 / 2}+(6.2 \pm 0.1) \cdot 10^{-6} T(\mathrm{~nm})$,

$(0.707<x<0.996 ; 298 \mathrm{~K}<T<1000 \mathrm{~K})$

$a\left(\mathrm{VN}_{x}\right)=0.38870+0.02488 x(\mathrm{~nm})$ at $T=298 \mathrm{~K}$.

$=$ lattice constant values for $\mathrm{NbN}_{x}$ from Langauer and Ettmayer. ${ }^{25}$ )

$=$ lattice constant values for $\mathrm{TaN}_{x}$ from Nie et al. ${ }^{26}$ )

than at the bottom surface being in contact with graphite spacer. It means that the $\mathrm{C}_{2}$ radical plume ${ }^{21)}$ with high carbon activity $a(\mathrm{C})$ and reactivity must have been emitted from the graphite lid downward to hit the test piece top surface.

In contrast to the results in the Group I experiments, those in the Group II experiments showed that the top surfaces are more highly nitrided than the bottom surfaces; compare (c) and (d) in Figs. 3-6. These evidences indicated that, in the test runs with no top graphite lid, target reaction for nitride formation was more closely achieved than in the condition with graphite lid. Anyway, the extent of carburization was considerable in the Group II experiments although not so much as in the Group I experiments.

So, as the next step, we decided to cut some frequency range of the solar beam before entering to the parabolic concentrator with a vague hope of suppressing $\mathrm{C}_{2}$ radical yield from the graphite crucible components.

Two filters supplied from Lee Filters (Hampshire, UK) were readily available at PROMES-CNRS; one was Sky blue filter (Ref.\# 068) and another Medium yellow filter (Ref.\# 010) with wave transmission performances as reproduced in Fig. 7. The spectral power distribution (SPD) curves displayed in Fig. 7 show the percentage of light at each wavelength that passes through the filter. As seen in Fig. 7, the Sky blue filter cuts more than $60 \%$ of the wave of wavelength between $500 \mathrm{~nm}$ and $700 \mathrm{~nm}$ while the Medium yellow filter cuts more than $80 \%$ of the wave of wavelength below $475 \mathrm{~nm}$.

The effect of the Sky blue filter towards retardation of carbide formation was quite impressive; (e) and (f) in Figs. 3-6. Wavelength of emission from $\mathrm{C}_{2}$ radical was reported to be $517 \mathrm{~nm}^{21)}$ which fell in the wavelength range between $500 \mathrm{~nm}$ and $700 \mathrm{~nm}$ that was cut efficiently with the Sky blue filter. We are still not very sure if there is some correlation between the suppressed wave transmission by the Sky blue filter and the emission wavelength from the $\mathrm{C}_{2}$ radical.

Compared with the Sky blue filter, effectiveness of the Medium yellow filter regarding the carburizing retardation 


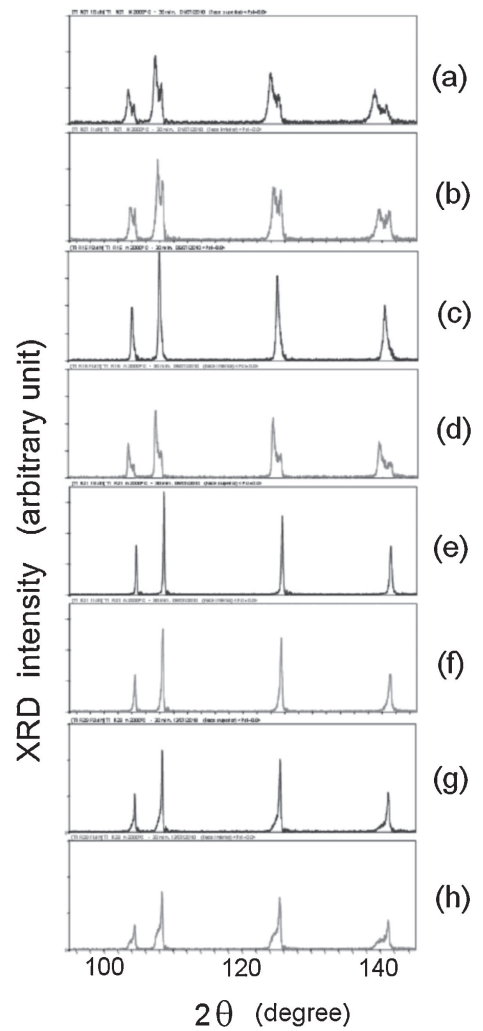

Fig. 3 XRD patterns for Ti test pieces over $2 \theta=95 \sim 145^{\circ}$. (a) Top surface/run 07, (b) bottom surface/run 07, (c) top surface/run 15, (d) bottom surface/run 15, (e) top surface/run 21, (f) bottom surface/run 21, (g) top surface/run 29, (h) bottom surface/run 29.

did not appear very impressive; (g) and (h) in Figs. 3-5. With the Medium yellow filter, more than $80 \%$ of the $517 \mathrm{~nm}$ wave was transmitted.

By insertion of either Sky blue or Medium yellow filter, measured pyrometric temperature drop at the monitoring spot was insignificant, on the contrary to our anticipation of detecting considerable pyrometric temperature drop. This evidence appeared to imply that the heating effect by concentrated solar beam must be largely dependent on intensity of the waves in IR (infra-red) regime rather than that of UV (ultra-violet) range.

As reported recently, ${ }^{10}$ in case of carbide synthesis starting from $\mathrm{G} / \mathrm{W}$ powder mixtures at hypo-stoichiometric composition, very top and very bottom surface of thickness $0.1 \mathrm{~mm}$ had to be considered as singular zone where the constitution of the phase was different from the rest of the intermediate bulk. Thus, to look into this aspect for the present set of test pieces, we undertook XRD characterization for some selected test pieces by grinding away $0.1 \mathrm{~mm}$ top surface layer.

\subsection{XRD patterns after removing $0.1 \mathrm{~mm}$ top layer}

This selective XRD characterization removing $0.1 \mathrm{~mm}$ thick top layer was done for the following test pieces; Ti (run 07) and $\mathrm{Nb}$ (run 17). The results are summarized in Fig. 8.

It is seen in Fig. 8 that, for both Ti (run 07 with the setup A with the graphite lid) and $\mathrm{Nb}$ (run 17 with the setup $\mathrm{B}$ without the graphite lid), the XRD peak on the higher $2 \theta$

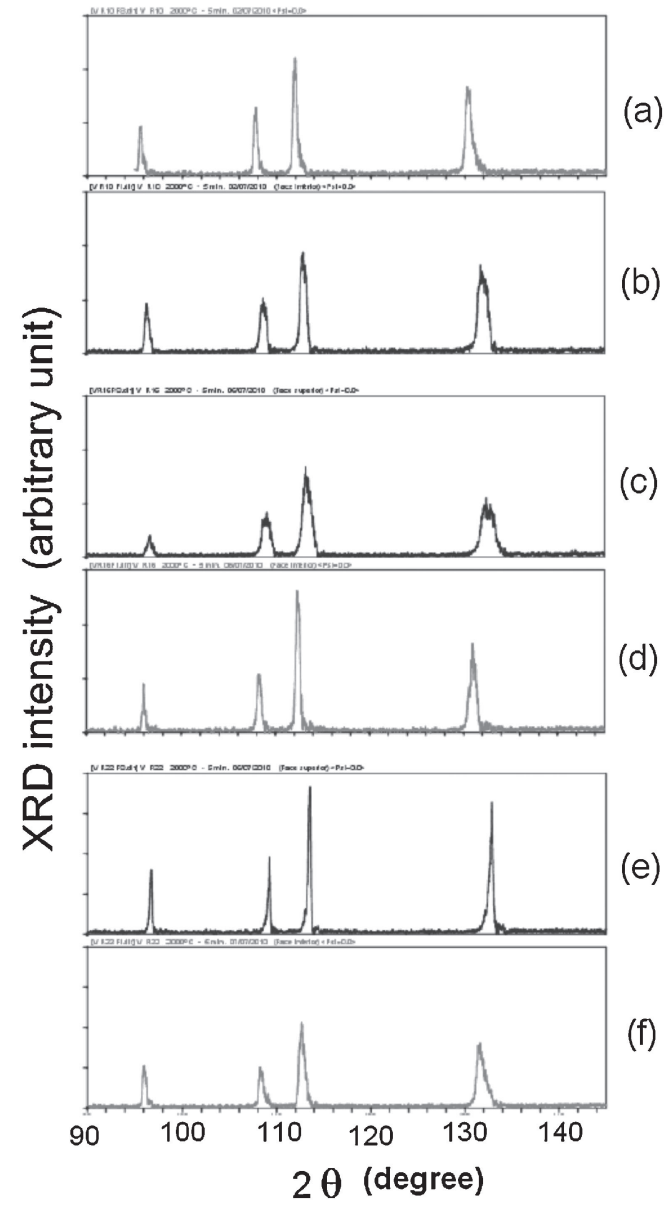

Fig. 4 XRD patterns for $\mathrm{V}$ test pieces over $2 \theta=90 \sim 145^{\circ}$. (a) Top surface/run 10, (b) bottom surface/run 10, (c) top surface/run 16, (d) bottom surface/run 16, (e) top surface/run 22, (f) bottom surface/run 22.

shoulder side of any given $(h k l)$ index intensified compared with the lower $2 \theta$ side suggesting lesser extent of carburization at the depth by $0.1 \mathrm{~mm}$ away from the top surface. Thus, the very top surface layer of the solar-processed carbide or carbo-nitride sample might better to be considered with certain degree of singularity irrespective of whether the starting material was pure $\mathrm{M}$ or $\mathrm{G} / \mathrm{M}$ mixture.

\subsection{Formation of tetragonal $\mathrm{Nb}_{4} \mathrm{~N}_{3}$ phase}

XRD patterns for the $\mathrm{Nb}$ test pieces at the top surface excluding the run 11 (Fig. 5(a)) showed line splitting (Fig. 5(c), (e) and (g); Fig. 8). The XRD patterns for the $\mathrm{Nb}$ test piece in the test run 11 showed that the formed phase was almost pure $f c c \mathrm{NbC}$ both at the top (Fig. 5(a)) and the bottom (Fig. 5(b)).

The detected XRD speak splitting in Fig. 5(c), (e) and (g) and also in Fig. 8 must be ascribed to the known phenomenon of tetragonal phase formation at around $x=0.75$ for $\mathrm{NbN}_{x} \cdot{ }^{27,28)}$

Under the experimental setup B in Fig. 1 with no filter (Group II experiment; run 17; Fig. 5(c)) and with Medium yellow filter (Group IV experiments; run 26; Fig. 5(g)), the XRD peak splitting looked more pronounced compared with that in the Group III experiments with Sky blue filter (run 26; Fig. 5(e)). 


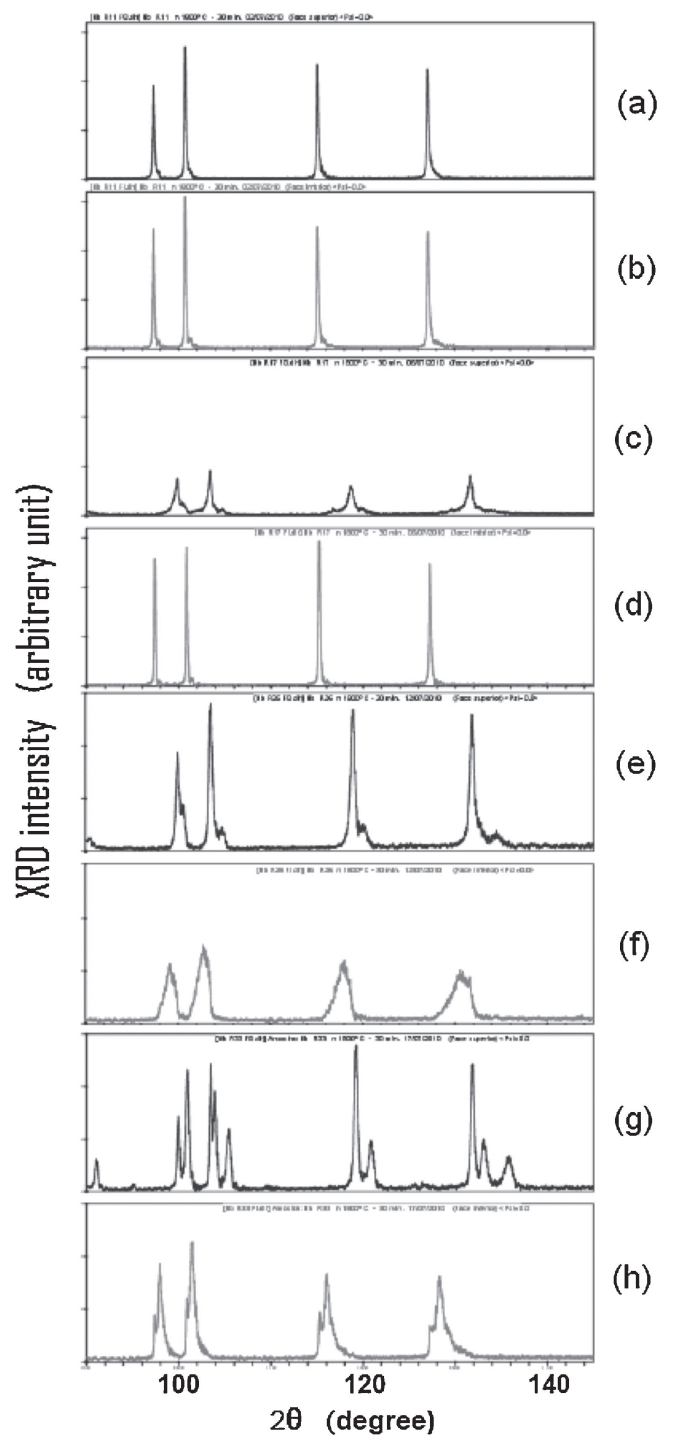

Fig. $5 \mathrm{XRD}$ patterns for $\mathrm{Nb}$ test pieces over $2 \theta=90 \sim 145^{\circ}$. (a) Top surface/run 11, (b) bottom surface/run 11, (c) top surface/run 17, (d) bottom surface/run 17, (e) top surface/run 26, (f) bottom surface/run 26, (g) top surface/run 33, (h) bottom surface/run 33.

Thus, the observed evidences appeared to suggest that the suppressed $\mathrm{C}_{2}$ radical yield with the Sky blue filter allowed higher $\mathrm{N}$ concentration in the $\mathrm{Nb}$ lattice to allow formation of $f c c \mathrm{Nb}(\mathrm{C}, \mathrm{N})$ rather than tetragonal $\mathrm{Nb}_{4} \mathrm{~N}_{3}$.

This effect of Sky blue filter towards suppression of $\mathrm{C}_{2}$ radical yield certainly deserves further experimental verification.

Similar case of tetragonal distortion of $f c c$ lattice was analyzed earlier for hypo-stoichiometric di-hydride $\mathrm{ZrH}_{2-z}$ of $\mathrm{Zr}$ at around $z \approx 0.3{ }^{29)}$

\section{5 $\mathrm{Ta}_{2} \mathrm{~N}$ phase formation}

In the standard XRD scan made for the Ta test pieces over $2 \theta$ range between $95^{\circ}$ and $145^{\circ}$, several additional lines that were not identifiable as $f c c \mathrm{Ta}(\mathrm{C}, \mathrm{N})$ appeared and the phase identification did not seem easy. To solve this doubt, $2 \theta$ scan was made over $30 \sim 80^{\circ}$ range for the Ta runs 18,23 and 32 . The results of the scan are summarized in Fig. 9. The phase identification from this low $2 \theta$ range XRD patterns was quite

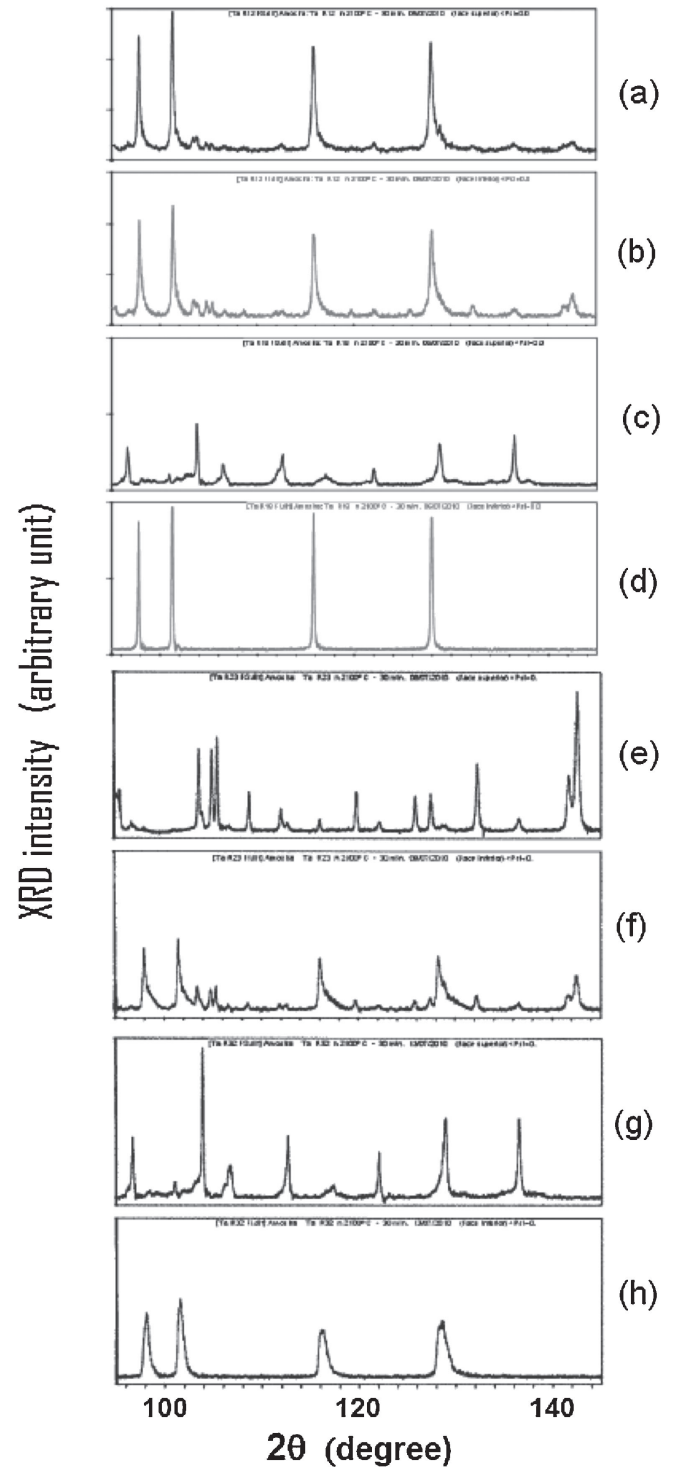

Fig. 6 XRD patterns for Ta test pieces over $2 \theta=95 \sim 145^{\circ}$. (a) Top surface/run 12, (b) bottom surface/run 12, (c) top surface/run 18, (d) bottom surface/run 18, (e) top surface/run 23, (f) bottom surface/run 23, (g) top surface/run 32, (h) bottom surface/run 32 .

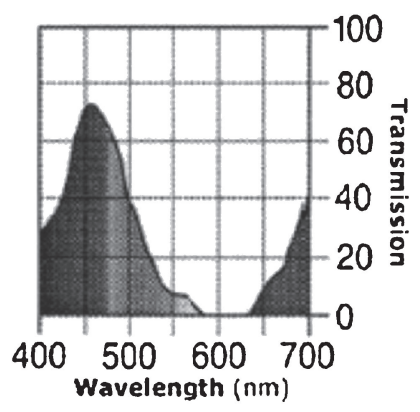

Sky Blue _ 068

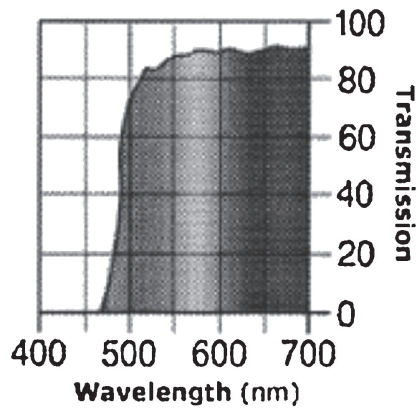

Medium Yellow_010
Fig. 7 Wave transmission performances of the used Lee Colour Filters. left: Sky blue (Ref.\# 068) filter; reproduced from http:// www.leefilters.com/lighting/products/finder/act:colourdetails/colourRef: C4630710C4A5A9/ right: Medium yellow (Ref.\# 010) filter; reproduced from http://www.leefilters.com/lighting/products/finder/act:colourdetails/ colourRef:C4630710C3E644/ 


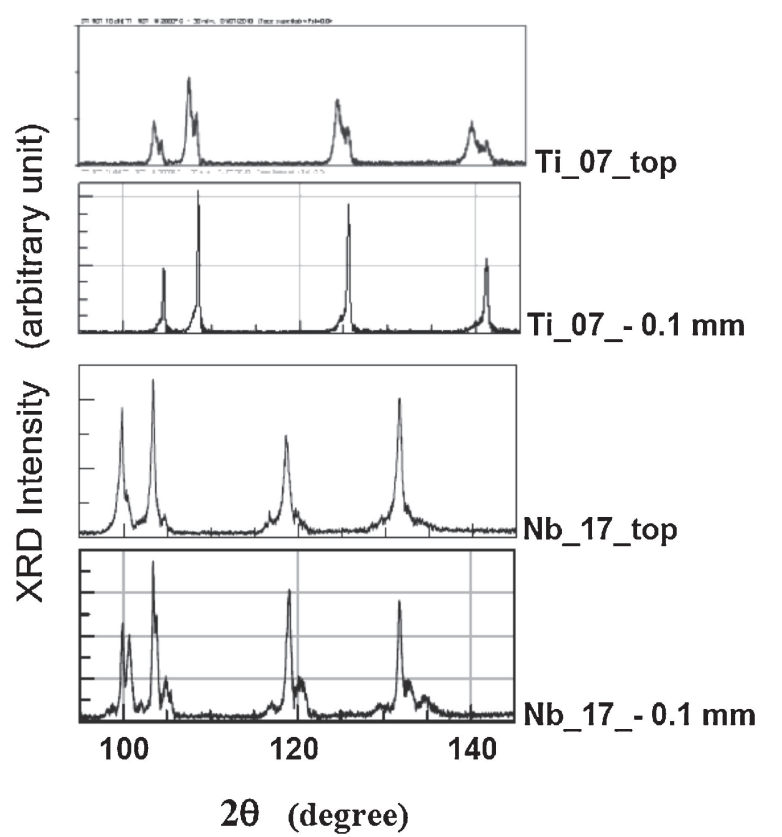

Fig. 8 XRD patterns over $2 \theta=95 \sim 145^{\circ}$ compared between the top surface and the depth at around $0.1 \mathrm{~mm}$ from the top layer for the specimens of $\mathrm{Ti}$ (run 7) and of $\mathrm{Nb}$ (run 17).

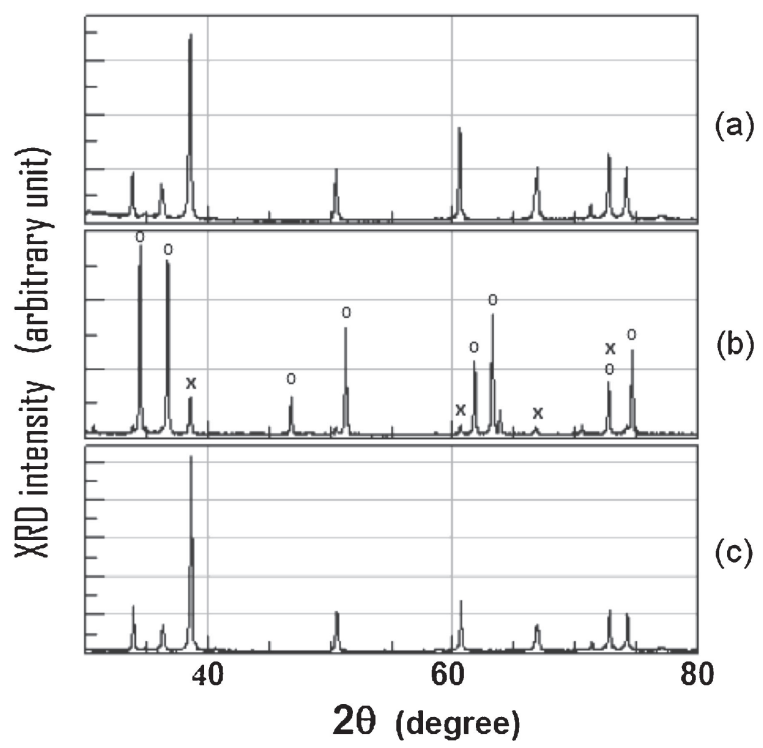

Fig. 9 XRD patterns for Ta test pieces over $2 \theta=30 \sim 80^{\circ}$ after removing ca. $0.1 \mathrm{~mm}$ top layer. (a) run 18 , (b) run 23 , (c) run 32. o: TaN (JCPDSICDD 03-065-1947), x: Ta 2 N (JCPDS-ICDD 00-026-0985).

simple by comparing with the files issued by JCPDS-ICDD (Joint Committee on Powder Diffraction Standards-International Centre for Diffraction Data); JCPDS-ICDD 03-0651947 for TaN and 00-026-0985 for $\mathrm{Ta}_{2} \mathrm{~N}$.

As seen in Fig. 9, TaN was the dominant phase in the run 23 (with Sky blue filter) while $\mathrm{Ta}_{2} \mathrm{~N}$ was dominant in the runs 18 (no filter) and 32 (Medium yellow filter). As such, like in the case with $\mathrm{Nb}$, higher nitride formation for Ta was promoted under irradiation with solar beam filtered through Sky blue filter.

In binary $\mathrm{Ta}-\mathrm{N}$ system, sub-nitride $\mathrm{Ta}_{2} \mathrm{~N}$ might have comparatively high stability like sub-carbide $\mathrm{Ta}_{2} \mathrm{C}$ in binary
Ta-C system. In our first carbide synthesis work at PROMESCNRS, we undertook experiment for Ta-C system and found $\mathrm{Ta}_{2} \mathrm{C}$ phase formed initially as the intermediate phase even under presence of excess free carbon while they are fully converted to thermodynamically stable $\mathrm{TaC}$ phase eventually after prolonged reaction duration. ${ }^{11)}$

\section{Conclusions}

Following conclusions were drawn from the present work attempting nitride formation for $\mathrm{Ti}, \mathrm{V}, \mathrm{Nb}$ and $\mathrm{Ta}$ in $\mathrm{N}_{2}$ gas environment under heating with concentrated solar beam.

(1) In solar furnace, sample holder is usually made of graphite on account of high thermal stability to withstand heating to a temperature exceeding $3000^{\circ} \mathrm{C}$. In this common setting, synthesis of pure nitride is not easy on account of yield of $\mathrm{C}_{2}$ radical with high $a(\mathrm{C})$ from the graphite sample holder and the reaction products would end up with carbonitride rather than nitride.

(2) Experimental evidences indicated that insertion of Sky blue filter efficiently retarded the carburizing process to lead to formation of phases with high $\mathrm{N}$ content although it remained unclear whether the retarded carburization is ascribed to suppressed emission of $\mathrm{C}_{2}$ radical or to direct influence of selective cut of certain wavelength range on the reaction mechanism itself.

(3) By insertion of either Sky blue filter or Medium yellow filter, detected pyrometric temperature drop was insignificant suggesting that heating effect in concentrated solar beam must be largely contributed from the wave in IR range rather than UV range.

(4) In the present nitriding experiments in $\mathrm{N}_{2}$ gas environment using graphite crucible carried out at $2000^{\circ} \mathrm{C}$, prepared carbo-nitride was largely homogeneous excluding only the very bottom layer unlike in the case of carbide synthesis for $\mathrm{W}$ undertaken in inert $\mathrm{Ar}$ gas environment at $1600^{\circ} \mathrm{C} .{ }^{10)}$

(5) Temperature measurement system must be improved to allow more precise temperature control for synthesis of reaction products whose phase relationships might vary sensitively with temperature.

\section{Acknowledgements}

Travel and subsistence costs for NS, FACO and LGR were subsidized by EC (European Commission) through SFERA (Solar Facilities for the European Research Area) programme with contract reference number SFERA-228296 (acronym SunSynt). Project officers, Ms Marie PROUTEAU and Dr. Gabriel OLALDE, at PROMES-CNRS are sincerely thanked for their very kind attention to make 3-weeks stay of NS, FACO and LGR in Font-Romeu as comfortable as possible. The authors would like to thank technical staff of PROMES-CNRS, especially Mr. Nicolas BOULLET and Mr. Emmanuel GUILLOT, for their diligent efforts to significantly improve the optical line-up and experimental setup of the solar furnace facilities. The authors also appreciate constant attention of Dr. Gilles FLAMANT, Director of PROMES-CNRS, to make the progress of the experimental work smooth. 


\section{REFERENCES}

1) J. Cruz Fernandes, L. Guerra Rosa, D. Martínez, J. Rodríguez and N. Shohoji: J. Ceram. Soc. Jpn. 106 (1998) 839-841.

2) D. Martínez, J. Rodríguez, L. Guerra Rosa, J. Cruz Fernandes and N. Shohoji: J. de Physique IV (Symposium Series), Vol. 9, Proceedings 3 (1999) pp. 405-410.

3) N. Shohoji, L. Guerra Rosa, J. Cruz Fernandes, D. Martínez and J. Rodríguez: Mater. Chem. Phys. 58 (1999) 172-176.

4) L. Guerra Rosa, J. Cruz Fernandes, P. M. Amaral, D. Martínez, J. Rodríguez and N. Shohoji: Int. J. Refractory Met. Hard Mater. (Successor to Planseeberichte für Pulvermetallurgie), 17 (1999) 351356

5) J. Cruz Fernandes, P. M. Amaral, L. Guerra Rosa, D. Martínez, J. Rodríguez and N. Shohoji: Int. J. Refractory Met. Hard Mater. 17 (1999) 437-443.

6) N. Shohoji, P. M. Amaral, J. Cruz Fernandes, L. Guerra Rosa, D. Martínez and J. Rodríguez: Mater. Trans., JIM 41 (2000) 246-249.

7) P. M. Amaral, J. Cruz Fernandes, L. Guerra Rosa, D. Martínez, J. Rodríguez and N. Shohoji: Int. J. Refractory Met. Hard Mater. 18 (2000) 47-53.

8) J. Rodríguez, D. Martínez, L. Guerra Rosa, J. Cruz Fernandes, P. M. Amaral and N. Shohoji: J. Solar Energy Eng. 123 (2001) 109-116.

9) J. Cruz Fernandes, C. Anjinho, P. M. Amaral, L. Guerra Rosa, J. Rodríguez, D. Martínez, F. Almeida Costa Oliveira and N. Shohoji: Mater. Chem. Phys. 77 (2002) 711-718.

10) N. Shohoji, T. Magalhães, F. Almeida Costa Oliveira, L. Guerra Rosa, J. Cruz Fernandes, J. Rodríguez, I. Cañadas and D. Martínez: Mater. Trans. 51 (2010) 381-388.

11) J. Cruz Fernandes, F. Almeida Costa Oliveira, B. Granier, J.-M. Badie L. Guerra Rosa and N. Shohoji: Solar Energy 80 (2006) 1553-1560.

12) F. Almeida Costa Oliveira, J. Cruz Fernandes, J.-M. Badie, B. Granier, L. Guerra Rosa and N. Shohoji: Int. J. Refractory Met. Hard Mater. 25 (2007) 101-106.

13) N. Shohoji, J.-M. Badie, B. Granier, F. Almeida Costa Oliveira, J. Cruz Fernandes and L. Guerra Rosa: Int. J. Refractory Met. Hard Mater. 25
(2007) 220-225.

14) F. Almeida Costa Oliveira, B. Granier, J.-M. Badie, J. Cruz Fernandes, L. Guerra Rosa and N. Shohoji: Int. J. Refractory Met. Hard Mater. 25 (2007) 351-357.

15) S. Dias, F. Almeida Costa Oliveira, B. Granier, J.-M. Badie, J. Cruz Fernandes, L. Guerra Rosa and N. Shohoji: Mater. Trans. 48 (2007) 919-923.

16) F. Almeida Costa Oliveira, B. Granier, J.-M. Badie, J. Cruz Fernandes, L. Guerra Rosa and N. Shohoji: Mater. Sci. Forum 587-588 (2008) 993-997.

17) B. Granier, J.-M. Badie, F. Almeida Costa Oliveira, T. Magalhães, N. Shohoji, L. Guerra Rosa and J. Cruz Fernandes: Mater. Trans. 49 (2008) 2673-2678.

18) B. Granier, N. Shohoji, F. Almeida Costa Oliveira, T. Magalhães, J. Cruz Fernandes and L. Guerra Rosa: Mater. Trans. 50 (2009) 28132819.

19) Y. S. Touloukian and D. P. Dewitt: Thermoradiative Properties of Nonmetallic Solids, Thermodynamic Properties of Matter, Vol. 8, (IFI/ Plenum, New York and Washington, 1972) p. 52.

20) M. W. Chase, Jr. (Ed.): NIST-JANAF Thermochemical Tables, Fourth Edition, J. Phys. Chem. Ref. Data, Monograph No. 9, (Am. Chem. Soc. Am. Inst. Phys., 1998).

21) J.-M. Badie, G. Flamant, T. Guillard and D. Laplaze: Chem. Phys. Lett. 358 (2002) 199-206

22) E. K. Storms: The Refractory Carbides, (Academic Press, New York, 1967).

23) S. Nagakura, T. Kusunoki, F. Kakimoto and Y. Hirotsu: J. Appl. Cryst. 8 (1975) 65-66.

24) W. Langauer and P. Ettmayer: Monatsh. Chemie 117 (1986) 713-719.

25) W. Langauer and P. Ettmayer: Monatsh. Chemie 117 (1986) 275-286.

26) H. B. Nie, S. Y. Xu, S. J. Wang, L. P. You, Z. Yang, C. K. Ong, J. Li and T. Y. F. Liew: Appl. Phys. A 73 (2001) 229-236.

27) E. C. Ethridge, S. C. Erwin and W. E. Pickett: Phys. Rev. B 53 (1996) 12563-12565.

28) R. Berger, W. Langauer and P. Ettmayer: J. Alloy. Compd. 259 (1997) L9-L13.

29) N. Shohoji and T. Marcelo: J. Mater. Sci. Lett. 6 (1987) 1251-1253. 